\title{
IMPROVEMENT OF POSITION TRACKING PERFORMANCE OF ELECTRO HYDRAULIC ACTUATOR SYSTEMS WITH DISTURBANCE
}

\author{
Min Gyu Park*, Sung Hwan Park *, Jong Shik Kim* and Ho Gil Lee** \\ * School of Mechanical Engineering, Pusan National Univeristy \\ Jangjeon-dong, Geumjeong-gu, Busan, 609-735, Korea \\ (E-mail: pmgking@naver.com) \\ ** Dong Jin Co., LTD \\ 10-6 Jukgock-li Jinyoung-up, Kimhea, Kyungnam, 621-801, Korea \\ (E-mail: hklee1@dongjin.com)
}

\begin{abstract}
Electro hydraulic actuator (EHA) system has inner and outer loops like a cascade system. The inner loop consists of an electric motor, a gear pump, and an angular velocity controller, and the outer loop consists of a hydro-actuator and a position controller. Especially, dead-band nonlinearity that exists between the electric motor and the gear pump and friction that occurs between the cylinder and the piston are considered. The tracking performance of EHA position control systems becomes unsatisfactory due to the dead-band and friction effects. Thus, in order to improve the position tracking performance of EHA systems with disturbance, backstepping control scheme for the desired position tracking is proposed, which is compared with the conventional PID scheme.
\end{abstract}

\section{KEY WORDS}

Electro Hydraulic Actuator, Backstepping control

\section{INTRODUCTION}

Hydraulic actuators, in general, have shortcomings such as the leakage of working fluid, environmental pollution due to the leakage, a maintenance load, and limited working space, etc. In addition, conventional hydraulic actuator(CHA) systems have some problems in case of applying to fighting aircrafts, since fighting aircrafts require light weight as well as the improvement their response performance and fault detection for self safety or backup system. Therefore, to overcome these problems, EHA systems have been studied for the integration of components and electric motor control. However, the EHA systems have some merits such as size, energy efficiency, and faster response due to high stiffness compared with CHA systems.
The flow rate and pressure in EHA systems are adjusted directly by the control of the velocity and rotation direction of servo motor connected directly to the bidirectional hydraulic pump. Therefore, the motor control in EHA systems is very important to obtain precise position control performance and velocity control performance of the piston. The servo motor of EHA systems takes an important role for position and velocity controls of the actuator. The position tracking performance of the actuator is greatly influenced by the load pressure due to external load and friction between piston and cylinder. To overcome these problems, Pachter et. al. proposed a robust adaptive control scheme which can guarantee good position tracking control performance, although the system is influenced by disturbance and perturbation of system parameters[1]. 
Kokotovic et. al. suggested an EHA system with inner loop for the speed control and outer loop for the position control and applied an adaptive control scheme to the inner loop [2]. Although they proposed various control schemes to cope with system uncertainties and disturbance, the establishment of the inner loop controller is technically difficult and it is very expensive to develop it.

In this paper, a bacstepping controller is proposed for the position control of the piston rod with disturbances such as the load exerted on the piston rod and the contact friction between the piston and the cylinder, which affect to the rotation of the servo motor. And the backstepping scheme is compared with a conventional PID scheme for EHA systems with disturbances. Computer simulations are executed in order to verity the effectiveness of the backstepping control scheme for the EHA system with disturbances.

\section{SYSTEM MODELING}

Figure 1 shows an EHA system, which consists of the electric servo motor and the bi-directional gear pump. The gear pump is rotated by the servo motor and then the flow rate is generated in the gear pump. The generated pressure by the flow rate makes a position change in the piston rod. And the movement direction of the piston is related to the rotation direction of the servo motor.

As shown in Fig. 1, the servo motor and the bi-directional gear pump are connected directly. Therefore, the servo motor and the gear pump of the EHA position control system can be represented as [3, 4]

$T_{p m}=J_{p m} \dot{\omega}+B_{p m} \omega_{p}+T_{D B}+C_{p}\left(p_{a}-p_{b}\right)$

where $J_{p m}, B_{p m}, C_{p}, T_{D B}$ and $\omega_{p}$ are the equivalent inertia, equivalent viscous friction, volumetric capacity of the pump, torque loss due to static friction, and angular velocity of the pump, respectively.

To derive the dynamics of EHA systems, Newton's second law is applied for the hydraulic piston. Then,

$$
\ddot{x}(t)=\frac{1}{M}\left[A p_{l}-F_{\text {fric }}(\cdot)\right] \text {. }
$$

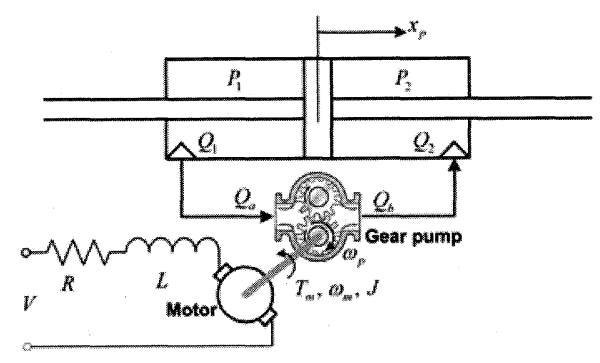

Figure 1 The schematic of an EHA system where $x(t), M, A, p_{l}$, and $F_{f r i c}$ are the moving position of piston, the piston mass, the cross section area of piston, the load pressure $\left(p_{a}-p_{b}\right)$, and LuGre friction, respectively. And the derivatives of pressures $p_{a}$ and $p_{b}$ of both the sides on piston can be represented as [5]

$\dot{p}_{a}=\frac{\beta_{k}\left(q_{a}-A \dot{x}\right)}{V_{a}}, \quad \dot{p}_{b}=\frac{\beta_{k}\left(q_{b}-A \dot{x}\right)}{V_{b}}$

The chamber volumes $V_{a}$ and $V_{b}$ depend on the position of the piston $x(t)$ as follows:

$V_{a}(t)=V_{0 a}+A x(t), V_{b}(t)=V_{0 b}+A x(t)$

The flow rates $\left(q_{a}, q_{b}\right)$ in the hydraulic pump can be represented with the pressure of the pump $\left(p_{a}, p_{b}\right)$ and the angular velocity $\left(\omega_{p}\right)$ of the pump as follows:

$q_{a}=C_{p} \omega_{p}-L_{f} p_{l}, \quad q_{b}=-q_{a}$

where $L_{f}$ is the leakage factor of the pump.

By differentiating Eq. (2), we can determine how these pressures can be controlled as follows:

$\dddot{x}=\frac{1}{M}\left[A \dot{p}_{l}-\dot{F}_{\text {fric }}\right]$

\section{CONTROLLER DESIGN}

Figure 2 shows the block diagram of the EHA position control system. The EHA position control system consists of the inner loop for the speed control of the servo motor and the outer loop for the position control of the piston. For the speed control of the motor in the inner loop, a proportional and derivative (PD) controller is used. In addition, the dynamics of the inner loop is not considered to design a backstepping position controller.

To design the backstepping controller, Eq. (6) is transformed as a general form [6] as follows:

$\dddot{x}=f+b u$

where

$$
\begin{aligned}
& f=-\frac{1}{M}\left[\beta_{k} \frac{A^{2}\left(V_{a}+V_{b}\right.}{V_{a} V_{b}} \dot{x}+\dot{F}_{f r i c}+L_{f} p_{L}\right], \\
& b=\frac{\beta_{k}}{M} \frac{A\left(V_{a}+V_{b}\right) C_{p}}{V_{a} V_{b}}, \quad u=\omega_{p} .
\end{aligned}
$$

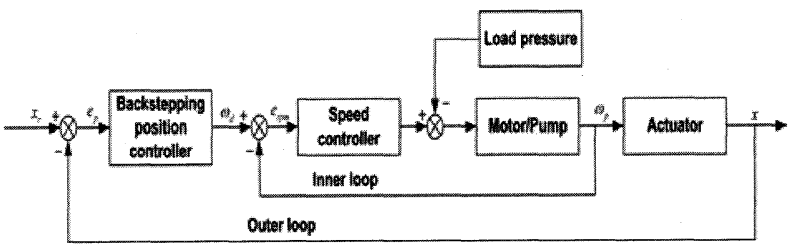

Figure 2 Block diagram of the EHA position control system 
Now, let us Eq. (2) represent state equations. Then,

$\left\{\begin{array}{l}\dot{x}_{1}=x_{2} \\ \dot{x}_{2}=x_{3} \\ \dot{x}_{3}=f+b u\end{array}\right.$

And, in order to design the backstepping controller, a new states are defined as follows:

$z_{1}=x_{1}-r(t)$

$z_{2}=x_{2}-\alpha_{1}\left(z_{1}\right)$

$z_{3}=x_{3}-\alpha_{2}\left(z_{1}, z_{2}\right)$

The design procedure of the backstepping controller is as follows:

\section{Step 1}

From Eq. (9), state equation for $z_{1}$ can be described as $\dot{z}_{1}=z_{2}+\alpha_{1}\left(z_{1}\right)-\dot{r}(t)$.

In order to select $\alpha_{1}\left(z_{1}\right)$ to guarantee stability for error dynamics in Eq. (12), the Lyapunov control function(LCF) is defined as

$V_{1}\left(z_{1}\right)=\frac{1}{2} z_{1}^{2}$.

Then,

$\dot{V}_{1}\left(z_{1}\right)=z_{1} \dot{z}_{1}=z_{1}\left[\alpha_{1}\left(z_{1}\right)-\dot{r}\right]+z_{1} z_{2}$.

From Eq. (14), if $\alpha_{1}\left(z_{1}\right)=-k_{1} z_{1}+\dot{r}$, Eq. (12) can be exponentially stable when $t \rightarrow \infty$. And $k_{1}>0$ is a design parameter.

\section{Step2}

From Eq. (10), state equation for $z_{2}$ can be described as

$\dot{z}_{2}=z_{3}+\alpha_{2}\left(z_{1}, z_{2}\right)-\dot{\alpha}_{1}\left(z_{1}\right)$

where

$$
\begin{aligned}
& \dot{\alpha}_{1}\left(z_{1}\right)=\frac{\partial \alpha_{1}}{\partial z_{1}} \dot{z}_{1}+\frac{\alpha_{1}}{\partial \dot{x}_{r}} \ddot{x}_{r} \\
& =-k_{1} \dot{z}_{1}+\ddot{x}_{r}=-k_{1}\left(z_{2}-k_{1} z_{1}\right)+\ddot{x}_{r} .
\end{aligned}
$$

Since Eq. (15) includes the information of Eq. (12), the LCF can be selected as

$$
V_{2}\left(z_{1}, z_{2}\right)=V_{1}\left(z_{1}\right)+\frac{1}{2} z_{2}^{2} \text {. }
$$

Then,

$$
\begin{aligned}
& \dot{V}_{2}\left(z_{1}, z_{2}\right)=\dot{V}_{1}\left(z_{1}\right)+\dot{z}_{2} z_{2} \\
& =-k_{1} z_{1}^{2}+z_{2} z_{3}+z_{2}\left[z_{1}+\alpha_{2}\left(z_{1}, z_{2}\right)-\dot{\alpha}_{1}\left(z_{1}\right)\right]
\end{aligned}
$$

If the last term of Eq. (17) is defined as

$z_{1}-\alpha_{2}\left(z_{1}, z_{2}\right)+\dot{\alpha}_{1}\left(z_{1}\right)=-k_{2} z_{2}$,

Then, $\alpha_{2}$ can be obtained as

$\alpha_{2}\left(z_{1}, z_{2}\right)=-\left(k_{1}+k_{2}\right) z_{2}-\left(1-k_{1}^{2}\right) z_{1}+\ddot{x}_{r}$

where $k_{2}>0$ is a design parameter.

Therefore,

$\dot{V}_{2}=z_{2} z_{3}-k_{1} z_{1}^{2}-k_{2} z_{2}^{2}$.

\section{Step 3}

From Eq. (11), the state equation for $z_{3}$ is described as $\dot{z}_{3}=\dot{x}_{3}-\dot{\alpha}_{2}\left(z_{1}, z_{2}\right)=(f+b u)-\dot{\alpha}_{2}\left(z_{1}, z_{2}\right)$

where

$$
\begin{aligned}
\dot{\alpha}_{2}\left(z_{1}, z_{2}\right)= & \frac{\partial \alpha_{2}}{\partial z_{1}} \dot{z}_{1}+\frac{\partial \alpha_{2}}{\partial z_{2}} \dot{z}_{2}+\frac{\partial \alpha_{2}}{\partial \dot{\alpha}_{1}} \ddot{\alpha}_{1} \\
& =-\dot{z}_{1}-k_{2} \dot{z}_{2}+\ddot{\alpha}_{1},
\end{aligned}
$$

and

$$
\begin{aligned}
\ddot{\alpha}_{1} & =\frac{\partial \alpha_{1}}{\partial z} \dot{z}_{1}+\frac{\partial \dot{\alpha}_{1}}{\partial z_{2}} z_{2}+\frac{\partial \dot{\alpha}_{2}}{\partial \ddot{x}_{r}} \ddot{x}_{r} \\
& =-k_{1}^{2} \dot{z}_{1}-k_{1} \dot{z}_{2}+\dddot{x}_{r} .
\end{aligned}
$$

Substituting Eqs. (12), (15), and (22) into Eq. (21), Eq. (21) can be rearranged as $\dot{\alpha}_{2}\left(z_{1}, z_{2}\right)=\left(k_{1}^{2}-1\right) \dot{z}_{1}-\left(k_{1}+k_{2}\right) \dot{z}_{2}+\dddot{x}_{r}$.

Since Eq. (20) uses the information of $z_{1}$ and $z_{2}$, the LCF included Eq. (16) can be described as

$V_{3}\left(z_{1}, z_{2}, z_{3}\right)=V_{2}\left(z_{1}, z_{2}\right)+\frac{1}{2} z_{3}^{2}$.

Then,

$$
\begin{aligned}
& \dot{V}_{3}\left(z_{1}, z_{2}, z_{3}\right)=\dot{V}_{2}+z_{3} \dot{z}_{3} \\
& =-k_{1} z_{1}^{2}-k_{2} z_{2}^{2}+z_{3}\left(z_{2}+f+b u-\dot{\alpha}_{2}\right) .
\end{aligned}
$$

If the last term of Eq. (24) is defined as

$-k_{3} z_{3}=z_{2}+f+b u-\dot{\alpha}_{2}\left(z_{1}, z_{2}\right)$,

From Eq. (25), the backstepping control law can be obtained as

$u=\frac{1}{b}\left(\dot{\alpha}_{2}-k_{3} z_{3}-z_{2}-f\right)$.

Substituting Eq. (25) into Eq. (24), Eq. (24) can be described as

$\dot{V}_{3}\left(z_{1}, z_{2}, z_{3}\right)=-k_{1} z_{1}^{2}-k_{2} z_{2}^{2}-k_{3} z_{3}^{2}$

Thus, from Eq. (27), the proposed backstepping control law is exponentially stable, since the time derivative of the LCF about new states is negative.

\section{SIMULATION AND DISCUSSION}

To design a backstepping position controller, only actuator dynamics neglected the inner dynamics is considered. And in order to confirm the position tracking performance of the proposed backstepping scheme, a conventional PID position controller is also designed. Figure 3 shows the position command input. The position command input $x_{r}(t)=0.05[\sin (0.4 \pi t)+\sin (2.4 \pi t)]$ has the maximum amplitude of $\pm 10[\mathrm{~cm}]$ and the variation of velocity during 5 seconds. The load pressure due to the friction between the piston and the cylinder is shown in Fig. 4. This load pressure interrupts the desired rotation of the servo motor and decreases the position tracking performance of the EHA position control systems.

From these simulation conditions, the position error results of the backstepping and PID control schemes are 
shown in Fig. 5. In case of the PID position control system, the pattern of the position error is a little irregular. However, that of the backstepping position control system is relatively regular. From this result, the backstepping position control system has desired robustness to the disturbance. Also, the transient response of the backstepping position control system is worse than that of the PID position control system. This result is why backstepping position controller use differential value of the position output state such as the velocity and the acceleration and excessive change of the initial error. The root mean square errors for the backstepping and the PID control systems are $0.019 \mathrm{~mm}$ and $0.08 \mathrm{~mm}$, respectively. Therefore, by using the backstepping scheme in place of the PID scheme, the tracking position performance of EHA system with disturbances can be improved by 4 times.

\section{CONCLUSION}

Position tracking performance of EHA systems with disturbances such as friction and dead-band are considered. In order to improve the position tracking performance of EHA with disturbances, backstepping

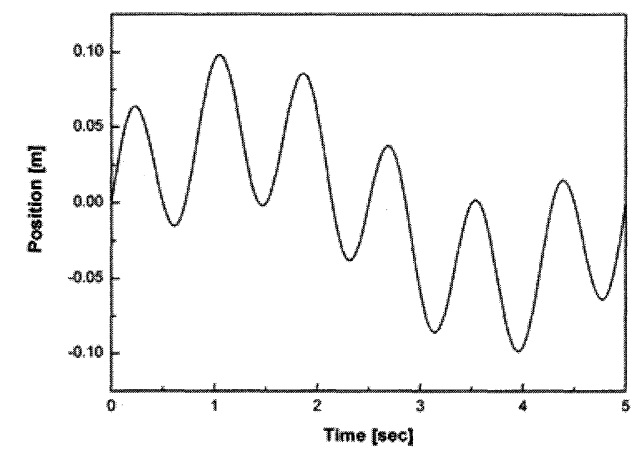

Figure 3 Position command input

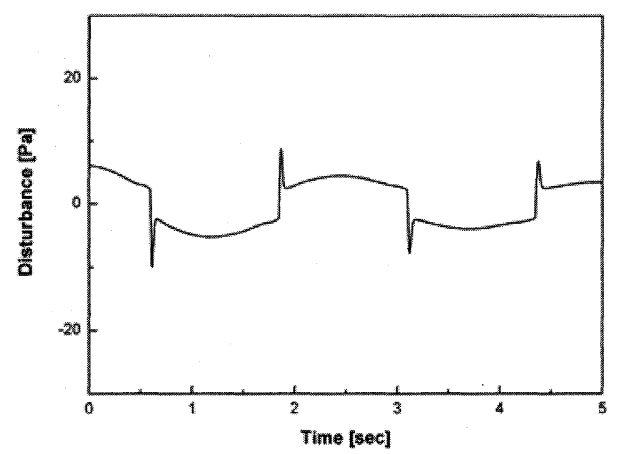

Figure 4 Disturbance input of the EHA system

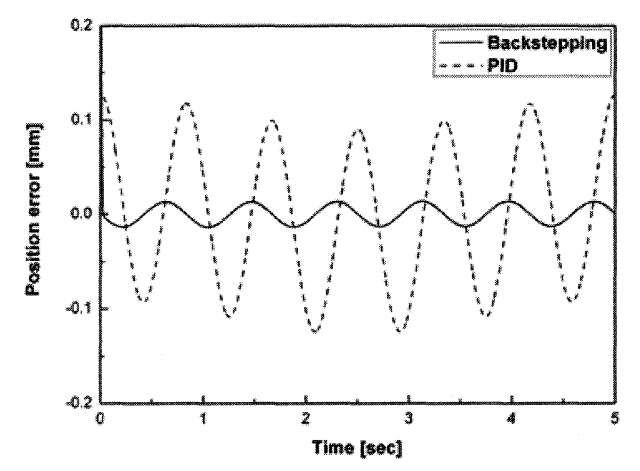

Figure 5 Position error of backstepping and PID control systems

position controller is proposed. It is found that backstepping control scheme based on the system model has robust characteristics for disturbances by computer simulation. The inner loop dynamics, which consist of a servo motor, a servo driver, and a speed controller, are considered as a total EHA systems. And the inner loop dynamics are not considered in a backstepping controller. However, the EHA position control system with the backstepping control scheme has desired position tracking performance and robustness.

\section{Acknowledgement}

This research was financially supported by the Ministry of Education, Science Technology (MEST) and Korea Industrial Technology Foundation (KOTEF) through the Human Resource Training Project for Regional Innovation.

\section{REFERENCES}

1. M. Pachter, C. H. Houpis, K. Kang, "Modeling and control of an electro-hydrostatic actuator", Int. J. of Robust and Nonlinear Control, 7(6), 591-608, 1997.

2. Vladimir V. Kokotovic, John Grabowski, Viral Amin and John Lee, "Electro Hydraulic Power Steering System", Int. Congress and Exposition Detroit, Michigan March, 1-4, 1999.

3. Watton, J., Fluid Power Systems, Prentice Hall, 1989.

4. Ogata, K., System Dynamics, $3^{\text {rd }}$ edition, Prentice Hall, 1998.

5. Habibi, S. and Goldenberg, A., A Mechatronics Approach for the Design of a New High Performance ElectroHydraulic Actuator, SAE, 1999, pp. 1-8.

6. Krstic, M., Kanellakopoulos, I. and Kokotovic, P., Nonlinear and Adaptive Control Design Wiley Interscience, New York, 1995. 